\title{
BOUNDING THE RISK OF CREW LOSS FOLLOWING ORBITAL DEBRIS PENETRATION OF THE INTERNATIONAL SPACE STATION AT ASSEMBLY STAGES 1J AND 1E
}

\author{
S. Evans', H. Lewis ${ }^{1}$, J. Williamsen ${ }^{2}$, H. Evans ${ }^{2}$, and W. Bohl ${ }^{2}$ \\ 'NASA Marshall Space Flight Center (MSFC), Huntsville, AL 35812, USA \\ ${ }^{2}$ University of Denver Research Institute (DRI), Denver, CO 80208, USA
}

\begin{abstract}
Orbital debris impacts on the International Space Station occur frequently. To date, none of the impacting particles has been sufficiently large to penetrate manned pressurized volumes. We used the Manned Spacecraft Crew Survivability code to evaluate the risk to crew of penetrations of pressurized modules at two assembly stages: after Flight $1 \mathrm{~J}$, when the pressurized elements of Kibo, the Japanese Experiment Module, are present, and after Flight 1E, when the European Columbus Module is present. Our code is a Monte Carlo simulation of impacts on the Station that considers several potential event types that could lead to crew loss. Among the statistics tabulated by the program is the probability of death of one or more crew members, expressed as the risk factor, $R$. This risk factor is dependent on details of crew operations during both ordinary circumstances and decompression emergencies, as well as on details of internal module configurations. We conducted trade studies considering these procedure and configuration details to determine the bounds on $\mathrm{R}$ at the $1 \mathrm{~J}$ and $1 \mathrm{E}$ stages in the assembly sequence. Here we compare the $\mathrm{R}$-factor bounds, and procedures and configurations that reduce $\mathrm{R}$ at these stages.
\end{abstract}

\section{MSCSUrv PROGRAM}

Orbital debris in Low Earth Orbit (LEO) poses a collision risk to active satellites, including the International Space Station (ISS). NASA has developed methods for modeling orbital debris hypervelocity impacts on the ISS, such as the BUMPER code. In addition to modeling impacts and penetrations, it is necessary to analyze the effects on crewmen and their operations after a penetration. To aid NASA in this goal, the University of Denver Research Institute (DRI) developed a computer program to specifically model impacts and quantify the damage risks to crew and spacecraft. The Manned Spacecraft and Crew Survivability (MSCSurv) code computes several statistics concerning results of penetrations of pressurized volumes, including the likelihood of crew loss. Results from hazard assessments performed with this program suggest modifications to planned crew operations during decompression emergencies to enhance crew safety.

\section{Processing Flow}

MSCSurv is a Monte-Carlo based simulation code, written in FORTRAN, and specifically developed to predict the ratio of penetrations leading to the loss of at least one crewmember to the total number of penetrations the risk factor, $\mathrm{R}$, for the manned modules of the ISS. In preparing for an MSCSurv run, the analyst must make assumptions regarding crew operations, crew vulnerability to hazardous conditions, hypervelocity impact phenomena, and other factors. Each of these assumptions carries an uncertainty, and MSCSurv calculates uncertainty bounds associated with the R-factors, based on the parameter uncertainties and distributions supplied by the analyst. Following a specified number of penetrations (typically $10^{6}$ ) using one set of parameter values, and comprising one simulation, MSCSurv varies input parameters and continues performing simulations. Experience has shown that a few hundred simulations (i.e., a few hundred million penetrations) may be required to develop 
reliable statistics. After running the required number of simulations, MSCSurv determines the mean value of each module's R-factor along with its associated two-standard-deviation (i.e., 2-sigma) uncertainty bounds, plus composite R-factors for the U.S. Segment, Russian Segment, and ISS as a whole.

A single pass through MSCSurv involves accessing the Impact Model, the Penetration and Damage Model, and the Spacecraft and Crew Loss Model. In the Impact Model a particle size, velocity, and approach direction are randomly selected based on the applicable orbital debris population model, which for our assessments was NASA's ORDEM96 (Kessler et al., 1996?). Next an impact location is selected randomly from those ISS elements exposed to particles from the selected approach direction, taking into account any shadowing by other ISS elements.

Having selected the particle and impact location, the flow proceeds to the Penetration and Damage Model, where the shield characteristics at the impact location are examined, and a determination made as to whether the particle could penetrate this element. If the shield ballistic limit indicates that the particle could not penetrate, processing returns to the Impact Model, and another particle, speed, and direction are drawn. If the particle does penetrate, damage equations are used to determine the hole and crack sizes expected to be produced by this penetration. The flow then passes to the Spacecraft and Crew Loss Model.

The Spacecraft and Crew Loss Model examines a cascade of damage possibilities; the determination of a loss at any of them sends the flow to the Loss Recording step without examining any less-catastrophic loss types. Loss types in the cascade are: 1) presence at the impact site of propellant tanks or other external equipment that could rupture and explosively depressurize a module they are attached to; 2) unbounded crack growth in the manned module pressure wall ("unzipping"); 3) destruction of critical internal equipment behind the point of impact; 4) destruction of payloads behind the point of impact, releasing toxic or high-energy substances in the vicinity of crew; 5) death due to hypoxia following the penetration; 6) crew injury due to fragments, pressure pulse, light flash, etc., associated with a nearby penetration; and 7) inability to depart in the escape vehicle due to generation of excessive ISS rotation rates by thrust of the gas venting from a penetration hole. Williamsen et al. (2001) provide more details of the analysis performed in the various models of the MSCSurv code.

\section{STUDY BACKGROUND}

This paper describes the R-factor analysis at two stages in the ISS assembly sequence, denoted IJ and $1 \mathrm{E}$. During the Space Shuttle Flight designated as $1 \mathrm{~J}$ the final pressurized elements of Kibo, the Japanese Experiment Module, are to be delivered, and on Flight $1 \mathrm{E}$ the European Columbus Module will be added. Modules present prior to these flights include the Service Module, FGB (Zarya), Universal Docking Module (UDM), Docking Compartment, Science Power Platform (SPP), Pressurized Mating Adapter-1 (PMA-1), Node 1, U.S. Laboratory, Airlock and High Pressure Gas Compartment, Node 2, PMA-2, and PMA-3. In addition, our models included a Progress resupply vehicle docked to the aft docking port of the Service Module, and a Soyuz crew return vehicle. For $1 \mathrm{~J}$ the Soyuz was docked at the nadir port of the Docking Compartment. For IE the Docking and Stowage Module, Node 3, PMA-3, and Cupola were added, and the Soyuz was docked to the Docking and Stowage Module's nadir port. Illustrations of the $1 \mathrm{~J}$ and $1 \mathrm{E}$ configurations are given in Figure 1.

The configuration data, including shield characteristics and geometries, was obtained from Prior (2000). Locations of internal equipment on the U.S. side were derived from Miller et al. (2000). Locations of internal equipment on the Russian side were adapted from Williamsen et al. (2001) and discussions with civil service and contractor personnel at Johnson Space Center (JSC) (Berdich, 2000, 2001; Lee, 2001).

Crew operations protocols were based on discussions with JSC personnel (Lamczyk, 2000, 2001; Alibaruho, 2000,2001 ), as well as emergency procedures documents prepared by NASA's Mission Operations Directorate (2001). A time of day was randomly selected for each penetration, and locations of crewmen assigned based on it. During the eight "sleep" hours, two crewmen were located in the aft portion of the Service Module and one in the U.S. Lab. During the sixteen "work" hours, crew locations were randomly selected based on frequency of module occupancy. Assumed occupancy times ranged from as few as seven minutes per day for infrequently-visited modules such as the SPP, to over four hours for the aft portion of the Service Module. 


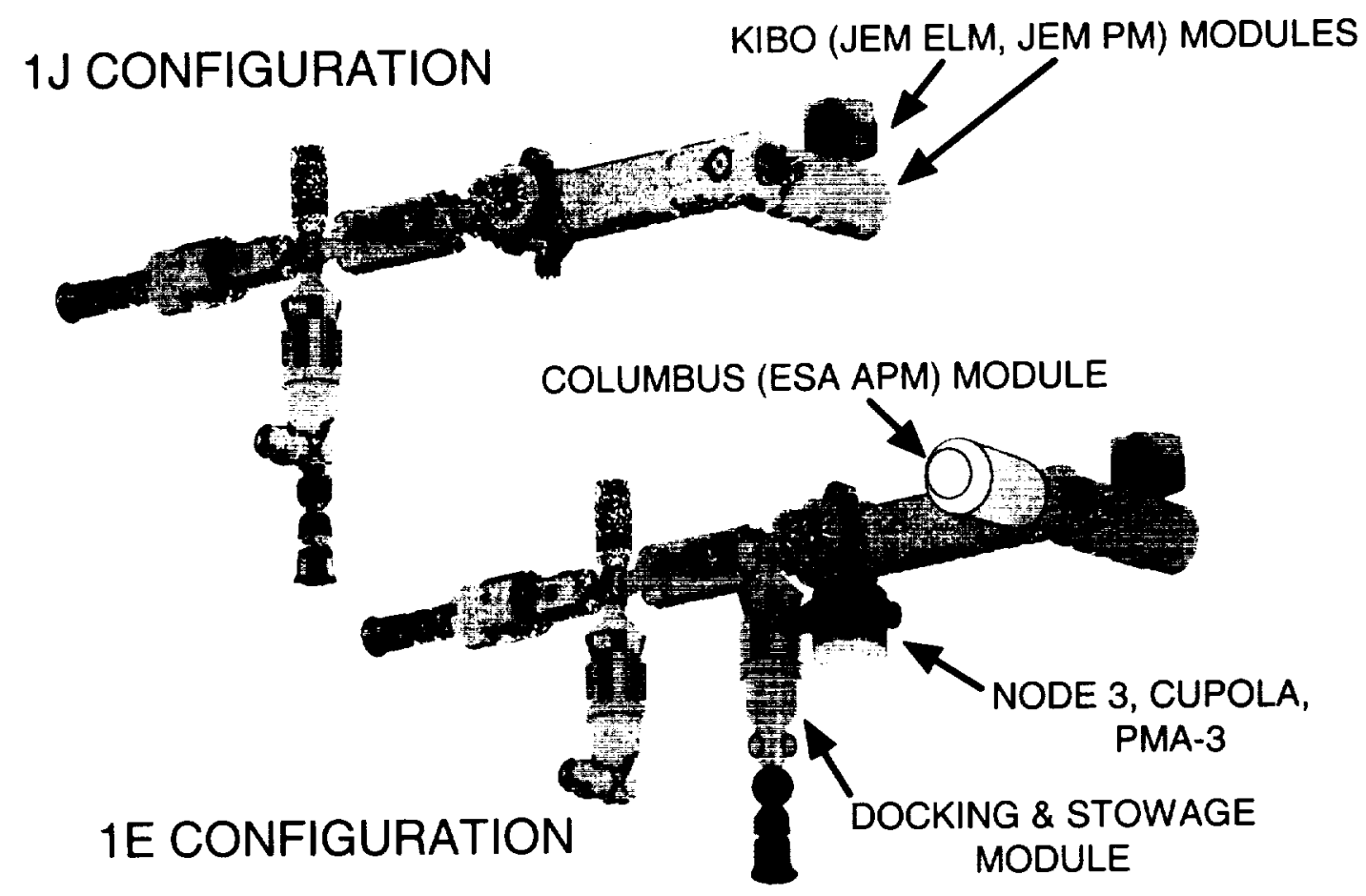

Fig. 1. International Space Station configurations used in this study.

\section{STUDY OBJECTIVES}

Objectives of this study were to:

1) Quantify the R-factor and its two-sigma upper and lower bounds for the $1 \mathrm{~J}$ and $1 \mathrm{E}$ configurations.

2) Study the effect of alternate crew procedures on R-factors.

3) Use R-factors in conjunction with Probability of No Penetration (PNP) from the BUMPER program analysis in Prior (2000) to estimate Probability of Crew Loss (PCL) at the $1 \mathrm{~J}$ and $1 \mathrm{E}$ stages.

\section{R-FACTOR COMPUTATION RESULTS}

\section{R-factors for Stage 1J - Baseline}

The R-factor results for the $1 \mathrm{~J}$ configuration, using baseline inputs, are given in Table 1 . The overall R-factor mean value of 0.250 indicates that approximately one in four penetrations of the station will lead to the death of one or more persons at Stage $1 \mathrm{~J}$.

Composite R-factors are determined by weighting each module's R-factor by its likelihood of penetration. At this assembly stage about $93 \%$ of penetrations occur on the Russian side, so the Russian R-factors drive the overall $\mathrm{R}$-factor. The NASA portion of the station tends to require larger particles to penetrate the shielding, so in the event of a penetration the hole sizes, crack lengths, and penetration depths tend to be larger. MSCSurv predicts a mean R factor of 0.408 for the NASA side of the station and 0.238 for the Russian side.

The largest contributor to the R-factor (i.e., most significant cause of death) on both sides of the station is hypoxia, accounting for $44.4 \%$ of the overall $\mathrm{R}$. Critical external equipment failure is the second leading contributor, accounting for $34.4 \%$ of total R. 


\begin{tabular}{|c|c|c|c|}
\hline Module & $2.275 \%$ & Mean & $97.725 \%$ \\
\hline $\begin{array}{l}\text { AIRLOCK } \\
\text { HPGC } \\
\text { JEM ELM } \\
\text { JEM PM } \\
\text { PMA_1 } \\
\text { PMA_2 } \\
\text { PMA_3 } \\
\text { NODE_1 } \\
\text { NODE_2 } \\
\text { US_LAB } \\
\text { FGB } \\
\text { PROG } \\
\text { SOYUZ } \\
\text { SPP } \\
\text { UDM } \\
\text { DK COMP } \\
\text { SERVFWD } \\
\text { SERVAFT }\end{array}$ & $\begin{array}{l}0.489 \\
0.063 \\
0.431 \\
0.350 \\
0.151 \\
0.172 \\
0.194 \\
0.183 \\
0.258 \\
0.430 \\
0.574 \\
0.142 \\
0.141 \\
0.006 \\
0.657 \\
0.031 \\
0.300 \\
0.108\end{array}$ & $\begin{array}{l}0.596 \\
0.072 \\
0.523 \\
0.481 \\
0.271 \\
0.336 \\
0.332 \\
0.304 \\
0.397 \\
0.567 \\
0.708 \\
0.261 \\
0.147 \\
0.010 \\
0.844 \\
0.080 \\
0.381 \\
0.151\end{array}$ & $\begin{array}{l}0.715 \\
0.080 \\
0.690 \\
0.682 \\
0.503 \\
0.573 \\
0.555 \\
0.498 \\
0.601 \\
0.732 \\
0.784 \\
0.330 \\
0.157 \\
0.014 \\
0.946 \\
0.103 \\
0.437 \\
0.283\end{array}$ \\
\hline $\begin{array}{l}\text { NASA } \\
\text { RSA }\end{array}$ & $\begin{array}{l}0.297 \\
0.182\end{array}$ & $\begin{array}{l}0.408 \\
0.238\end{array}$ & $\begin{array}{l}0.587 \\
0.301\end{array}$ \\
\hline ISS & 0.192 & 0.250 & 0.316 \\
\hline
\end{tabular}

Nonfatal injuries, loss of station attitude control, and Service Module depressurization all require abandonment of the station by the crew. These outcomes are only tallied by MSCSurv when no lethal failure mode occurs. Including these outcomes, our baseline runs predict that on average $58.5 \%$ of penetrations will result in either one or more deaths or a non-fatal event requiring evacuation of the crew, with a \pm two-sigma confidence interval of 0.530 to 0.634 .

\section{R-factors for Stage 1J - Modified Procedures}

In addition to analyzing the baseline case, we performed analyses to examine the effects of modifications to crew emergency procedures. The baseline rules call for the crew to gather in the Service Module on warning of a depressurization event, then proceed to the Soyuz to check its integrity. Only if the Soyuz is undamaged and pressure and pressure rate readings indicate at least 30 minutes remain before station cabin pressure reaches a "critical" value ( $\sim 60 \mathrm{~mm} \mathrm{Hg} \mathrm{-} \mathrm{"bail} \mathrm{pressure")} \mathrm{does} \mathrm{the} \mathrm{crew} \mathrm{egress} \mathrm{the} \mathrm{Soyuz} \mathrm{and} \mathrm{attempt} \mathrm{to} \mathrm{isolate} \mathrm{the} \mathrm{leak.}$ Although ISS has several Portable Breathing Apparatus (PBA) stations, donning these is not called for in the depressurization emergency rules.

Our procedure modifications included: 1) having the crew don PBA's upon warning of a depressurization event; 2) having the crew PBA's and isolate holes when the holes are heard (a minimum hole size of at least $2 \mathrm{~cm}$ is needed, and someone must pass the hole on their way through the affected module) even prior to Soyuz checkout; 3) having the crew isolate holes when they are heard even prior to Soyuz checkout (no PBA's); 4)ordered hatch closure, using only a bail pressure (not bail time) of $510 \mathrm{~mm} \mathrm{Hg}$ as a stopping condition; and 5) isolate holes upon hearing even prior to Soyuz checkout and use only the bail pressure as a bail condition.

Table 2 presents the mean ISS R-factors for each trade study. Donning PBA's, with no other changes to baseline assumptions, decreases hypoxia deaths dramatically (hypoxia decreased from $11.1 \%$ to $4.0 \%$ ), giving a decline in $\mathrm{R}$ from 0.250 to 0.182 . When the crew is using PBA's and isolating holes heard prior to Soyuz checkout, hypoxia increased by about $1 \%$ and crew departures decreased by about $2 \%$ compared to when the crew is using PBA's, but not isolating holes prior to Soyuz checkout. Early isolation alone or using a lower bail pressure result in larger R's. 
Table 2. Comparison of R-factors from baseline operations rules and various altered rules, $1 \mathrm{~J}$ configuration

$\begin{array}{rccccc}\text { Baseline } & \text { PBA's } & \text { PBA's + Early Isol. } & \text { Early Isol. } & \text { Bail at } 510 & \text { Early Isol. + Bail at } 510 \\ \text { Overall R: } 0.250 & 0.182 & 0.193 & 0.258 & 0.268 & 0.278 \\ \text { Hypoxia R: } 0.111 & 0.040 & 0.050 & 0.115 & 0.125 & 0.137\end{array}$

\section{R-factors for Stage 1E - Baseline}

As mentioned above, several modules were added to the $1 \mathrm{~J}$ configuration, or moved, to give the configuration at $1 E$. In addition, work day occupancy times were revised to include the new modules. Baseline R-factor results for $1 \mathrm{E}$ are given in Table 3. The overall mean $\mathrm{R}$-factor has increased slightly from the $1 \mathrm{~J}$ value to 0.277 . The $\mathrm{R}$ factor for the NASA side is down slightly, to 0.379 , while the Russian side value has increased slightly, to 0.267 .

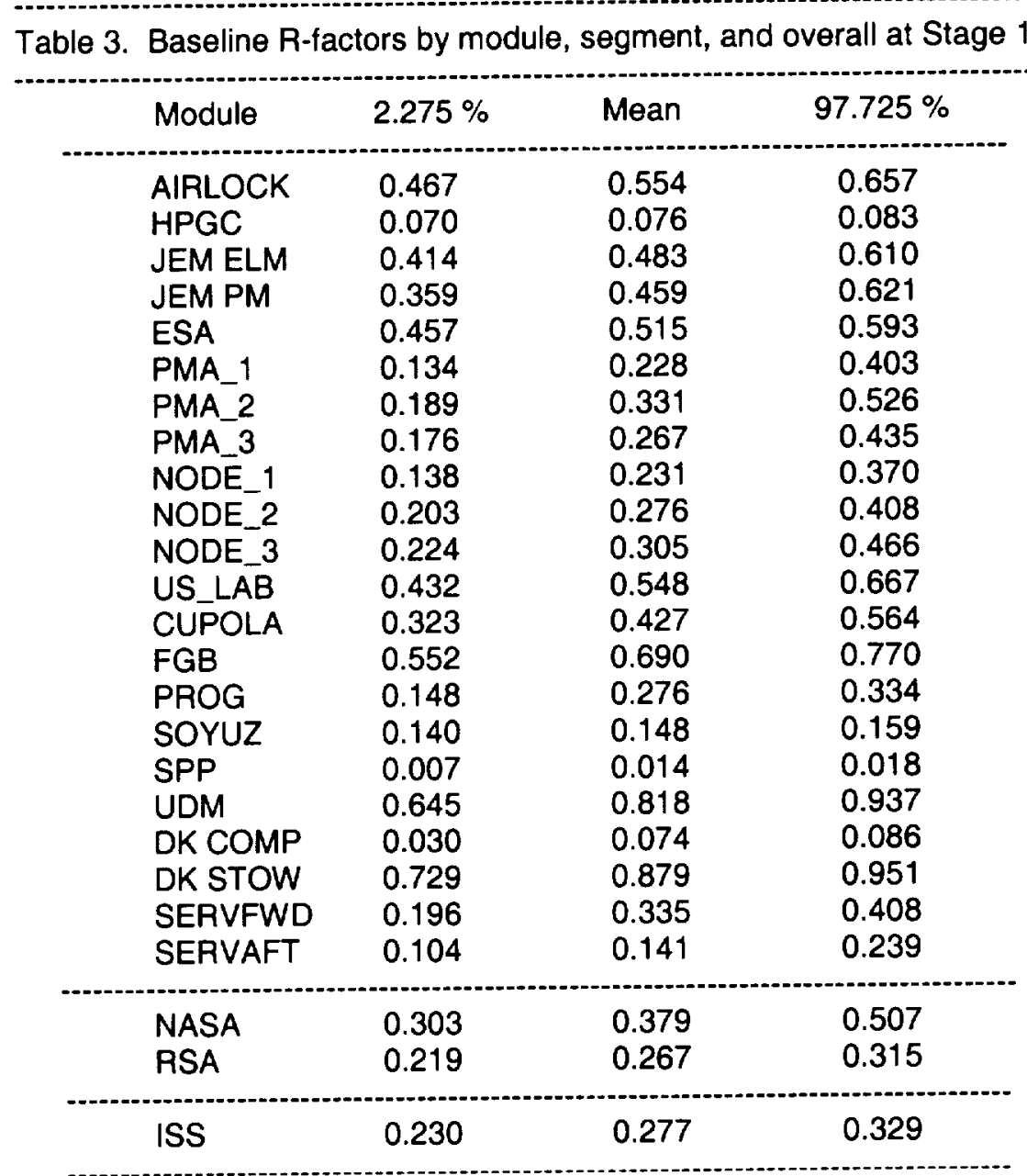

At Stage IE about $91 \%$ of all penetrations occur on the RSA side, down from $93 \%$ at $1 \mathrm{~J}$, presumably because several large modules have been added to the NASA segment, at the front of the station. Again, the largest contributor to $\mathrm{R}$ is hypoxia, at $42.6 \%$, and critical external equipment failure is second at $35.4 \%$ of $\mathrm{R}$. The added RSA module, Docking and Stowage, has high individual R's for both external equipment and hypoxia. The presence of this module in an exposed position increased the composite $\mathrm{R}$ for the station.

\section{R-factors for Stage 1E - Modified Procedures}

We repeated the alternative procedure trades discussed above for the $1 \mathrm{E}$ configuration, with R-factor outcomes given in Table 4 . 
Table 4. Comparison of R-factors from baseline operations rules and various altered rules, $1 \mathrm{E}$ configuration

$\begin{array}{lccccc}\text { Baseline } & \text { PBA's } & \text { PBA's + Early Isol. } & \text { Early Isol. } & \text { Bail at 510 } & \text { Early Isol. + Bail at 510 } \\ \text { Overall R: } 0.277 & 0.194 & 0.192 & 0.275 & 0.294 & 0.308 \\ \text { Hypoxia R: } 0.118 & 0.032 & 0.033 & 0.121 & 0.138 & 0.150\end{array}$

As in the $1 \mathrm{~J}$ case, donning PBA's dramatically improved survivability, and isolating modules where a hole is heard, prior to congregating at the Soyuz, also helped. Attempts at either isolating heard holes or using an ordered hatch closure protocol, without use of PBA's, either did not improve survival or impared it

\section{PROBABILITY OF CREW LOSS}

The final calculations determined the Probability of Crew Loss (PCL) for the ISS due to orbital debris penetrations at each of the $1 \mathrm{~J}$ and $1 \mathrm{E}$ stages. PCL can be calculated as

$$
\mathrm{PCL}=1-\mathrm{PNP}^{\mathrm{R}}
$$

where PNP is the Probability of No Penetration during the time span of interest. ISS is expected to be in the 1J configuration for a span of approximately 0.46 year during $2003-2004$, and in the 1E configuration for about one year beginning in 2004. PNP calculations were obtained from the BUMPER code; we associate no uncertainties with its output. Bounded PCL's for baseline operations rules and for use of PBA's upon depressurization warning are given in Table 5 .

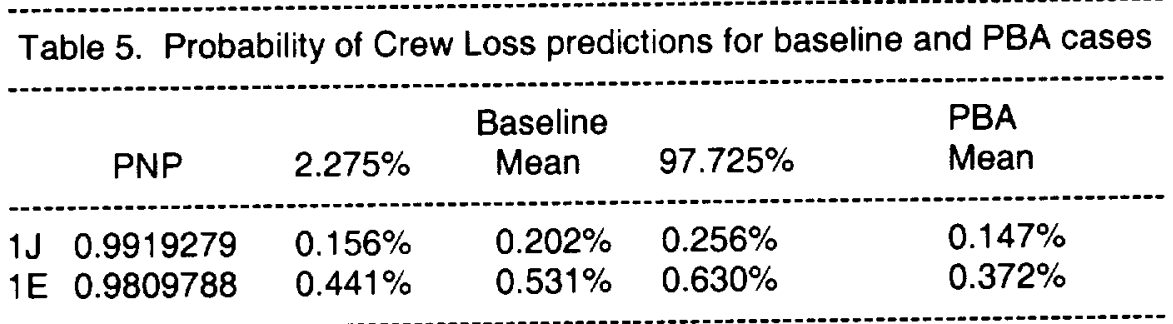

\section{REFERENCES}

Alibaruho, Q., private communications, February 2000 and August 2001.

Berdich, M., private communications, February 2000 and August 2001.

Kessler, D. J., J. Zhang, M. J. Matney et al., A Computer Based Orbital Debris Model for Spacecraft Design and Observation in Low Earth Orbit, NASA Technical Memorandum TM 104825, November 1996.

Lamczyk, P., private communications, February 2000 and August 2001.

Lee, R., private communication, August 2001.

Miller, J., L. Sorge, K. Fields, ISS Rack Traffic and On-Orbit Topology Handbook, Draft 6/00 Rev E Assembly Sequence, International Space Station Program Office, June 2000.

Mission Operations Directorate, Operations Division, International Space Station Complex Operations Emergency Procedures - All Expedition Flights, JSC 48512-7A, Johnson Space Center, June, 2001.

Prior, T., International Space Station Meteoroid \& Orbital Debris Integrated Threat Assessment \#9, JSC 29063, Lockheed Martin Space Operations, October, 2000.

Williamsen, J. E., H. J. Evans, W. E. Bohl, et al., Hazard Assessment Following Orbital Debris Penetration of International Space Station Manned Modules, Stage 12A Configuration, University of Denver Research Institute, Denver, CO, May 2001

E-mail address of S. Evans: steve.evans@msfc.nasa.gov

Manuscript received 19 October 2002; revised ; accepted 